\title{
Evaluation of Left Ventricular Function in Polymyositis and Dermatomyositis Patients with Global Longitudinal Strain Using Two Dimensional Speckle Tracking Methods
}

\author{
Elham Rajaei ${ }^{1}$, Nehzat Akiash ${ }^{2,}$, Karim Mowla ${ }^{3}$, Nasibeh Akiash ${ }^{4}$ and Shirin Azizidoost ${ }^{5}$ \\ ${ }^{1}$ Associated Professor of Rheumatology, Department of Rheumatology, Golestan Hospital, Ahvaz Jundishapur University of Medical Sciences, Ahvaz, Iran \\ ${ }^{2}$ Assistant Professor of Cardiology, Fellowship of Echocardiography, Atherosclerosis Research Center, Ahvaz Jundishapur University of Medical Sciences, Ahvaz, Iran \\ ${ }^{3}$ Associated Professor of Rheumatology, Department of Rheumatology, Golestan Hospital, Ahvaz Jundishapur University of Medical Sciences, Ahvaz, Iran \\ ${ }^{4}$ Internist, Department of Rheumatology, Golestan Hospital, Ahvaz Jundishapur University of Medical Sciences, Ahvaz, Iran \\ ${ }^{5}$ Research Assistant Professor of Clinical Biochemistry, Atherosclerosis Research Center, Ahvaz Jundishapur University of Medical Sciences, Ahvaz, Iran
}

* Corresponding author: Nehzat Akiash, Atherosclerosis Research Center, Ahvaz Jundishapur University of Medical Sciences, Ahvaz, Iran. Tel:+986132228037; Email: akiash.n@gmail.com

Received 2021 February 09; Revised 2021 June 26; Accepted 2021 July 11.

\begin{abstract}
Background: Heart problems are one of the main causes of death in patients with idiopathic inflammatory myopathies, such as polymyositis (PM) and dermatomyositis (DM). Speckle tracking echocardiography (STE) and global longitudinal strain (GLS) are noninvasive, accurate, repeatable, and angle-independent imaging techniques that facilitate a full global and regional evaluation of the left ventricular (LV) function.

Objectives: This study aimed to evaluate myocardial dysfunction in DM and PM patients using STE and GLS methods.

Methods: The present control-case study was conducted on 30 polymyositis and dermatomyositis patients and 40 healthy individuals as the control group. Both groups showed no symptoms of cardiovascular diseases and underwent two-dimensional STE and GLS evaluation. The GLS value was taken as a marker of LV systolic dysfunction.

Results: The 2D GLS value of LV was significantly lower in the DM and PM patients, compared to the control group. A mild diastolic dysfunction was observed in seven (23.3\%) patients, and $23(76.7 \%)$ patients had a normal state. The patients' age and duration of the disease were found to be significantly correlated with the left ventricular diastolic dysfunction. No significant difference was observed between the DM and PM patients with the control group in terms of pulmonary artery pressure level.

Conclusion: Although the DM and PM patients had normal left ventricular ejection fraction values, there was a significant difference between them and individuals in the control group in terms of LV dysfunction using GLS. Therefore, GLS is a useful variable that can be used to diagnose sustained and subclinical disorders in LV systolic function of DM and PM patients.
\end{abstract}

Keywords: Dermatomyositis, Diagnosis, Echocardiography, Imaging, Polymyositis

\section{Background}

Idiopathic inflammatory myopathies (IIM), including polymyositis (PM) and dermatomyositis (DM) are a group of muscular autoimmune diseases associated with considerable mortality and morbidity rates (1-3). These myopathies are diagnosed with proximal muscular atrophy, increased musculoskeletal enzymes, diagnostic electrocardiogram (ECG) findings, and muscle biopsy (4-6). Although the PM symptoms develop over several months, the diagnostic process is usually delayed since unlike DM it is not associated with skin lesions that appear before the muscles are affected (7).

Cardiac events in IIM patients are a major cause of death that mostly occur as a left ventricular (LV) diastolic disorder and are closely associated with the duration of the disease. Symptomatic heart problems are usually not common in these patients. The prevalence and importance of subclinical cardiac abnormalities in IIM have not yet been identified accurately. Moreover, autopsy findings indicate the presence of myocarditis in a group of these patients (8). Myocardial fibrosis increases the risk of systolic and diastolic dysfunction and dysrhythmia in these patients (9). The diagnosis of such cardiac diseases is often difficult with routine imaging techniques (10). Today, the development of non-invasive and highly sensitive diagnostic techniques has enabled the detection of subclinical cardiac abnormalities $(8,11)$.

Speckle tracking echocardiography (STE) is an echocardiography imaging technique that enables the objective and quantitative global and regional evaluation of myocardial systolic and diastolic myocardial functions $(12,13)$. Given that cardiac problems are poor prognostic signs in autoimmune disorders, studies have shown that LV deformation analysis using sensitive STE is appropriate for the prediction of subclinical LV myocardial involvement, even when ejection fraction (EF) is normal (14). The results of a recent study conducted by Diniz et al. showed that the application of two-dimensional STE (2D STE) led to the early detection of LV systolic myocardial impairment in juvenile DM patients with preserved EF (15).

Cardiac dysfunction, especially LV dysfunction, is one of the main causes of heart failure in DM/PM patients and often has a poor prognosis. Cardiac 
function assessment in patients with no known heart problems seems to be essential for their recovery and increases the chance of survival (16). Global longitudinal strain (GLS) is a new and more sensitive echocardiography method for the evaluation of LV function. Furthermore, a recent study has reported GLS as an important reproducible method for the measurement of $\mathrm{LV}$ function in comparison to LVEF (17).

\section{Objectives}

Regarding the small number of studies on the simultaneous evaluation of cardiac dysfunction and inflammatory myopathies, screening with novel imaging techniques, such as GLS and STE may facilitate the early diagnosis of subclinical LV dysfunction in inflammatory myopathies. Due to the significance of GLS and STE values in the assessment of LV dysfunction and considering the risk of cardiovascular diseases in inflammatory myopathies and the clinically asymptomatic cardiac dysfunctions in these patients, the present study was conducted to assess LV function using 2D STE and GLS evaluation as a non-invasive method to evaluate myocardial dysfunction and subclinical involvement in these patients.

\section{Methods}

The present case-control study was conducted over a period of six months. The study was conducted on 30 DM/PM patients over the age of 18 whose disease lasted over three years and had no history of known cardiovascular diseases, as well as 40 healthy people as the group of controls, who were matched with patients in terms of age and gender. All DM/PM patients who had been referred to rheumatology polyclinics of Ahvaz Jundishapur University of Medical
Sciences, Ahvaz, Iran, were contacted over a period of six months and were included in the study after the submission of written informed consent. Patients with a history of cardiac diseases, such as severe valvular problems, myocardial infarction, cardiomyopathy, and cardiac surgery were excluded from the study. Other exclusion criteria included the presence of abnormal signs in the patient's ECG and chest X-ray, the existence of heart murmurs or abnormal sounds in the heart examination, and the absence of a proper 2D view for the GLS evaluation. The study protocol was approved by the Ethics Committee of Ahvaz Jundishapur University of Medical Sciences, Ahvaz, Iran (IR.AJUMS.REC.1396.1004).

\subsection{Group allocation}

All the participants underwent full rheumatology and cardiovascular examination and their disease were diagnosed by a rheumatologist. Data related to the patients' risk factors, including age, gender, blood pressure, blood lipids, diabetes, smoking, family history, used medications, disease symptoms, systemic problems, and duration of the disease were collected from their records using a checklist. The control group was selected from among healthy people with no history of an underlying disease and cardiac or rheumatological problems. The age range in the control group was 20-60 years, and participants were selected voluntarily from among teaching hospitals' personnel and the patients' relatives.

Demographic information of the participants was acquired and clinical examinations and ECG were carried out on all participants in both control and case groups. Subsequently, data in both groups were compared. Figure 1 shows a diagram of the study progress.

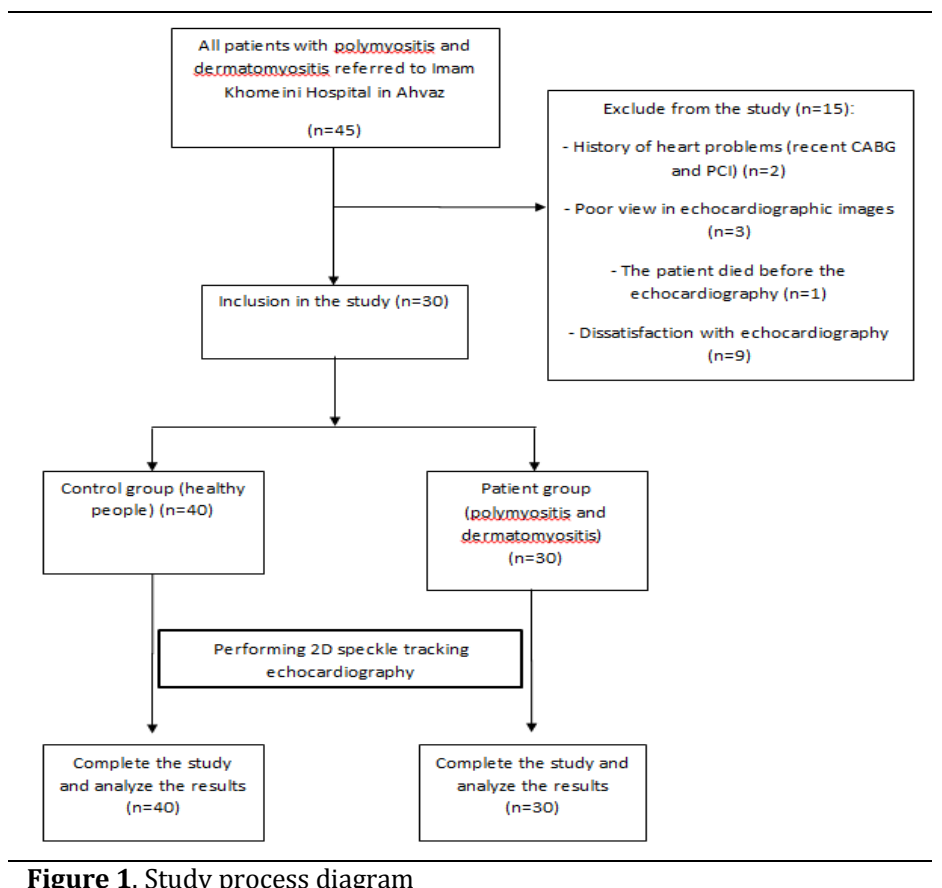




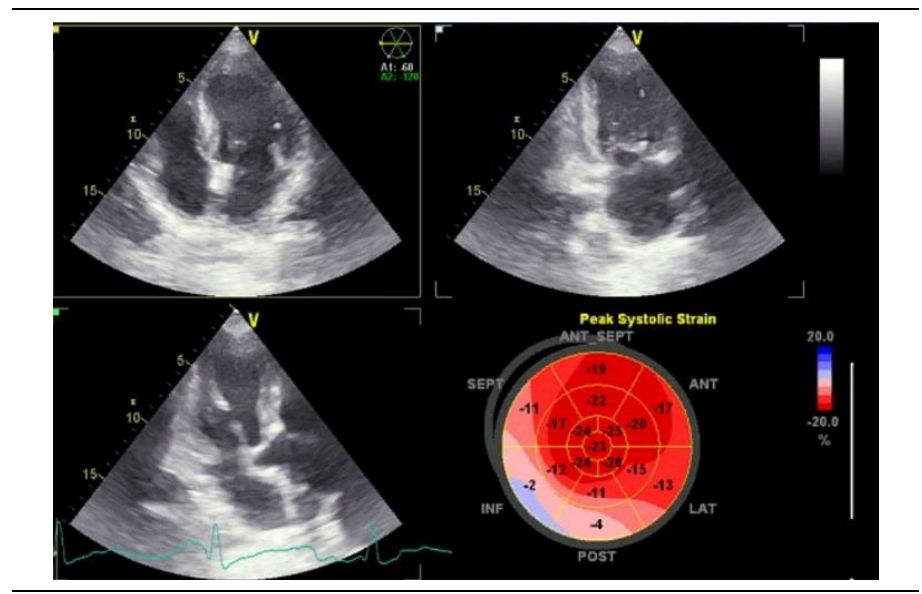

Figure 2. Three standard views, two-, three- and apical four-chamber views for evaluation of Longitudinal strain

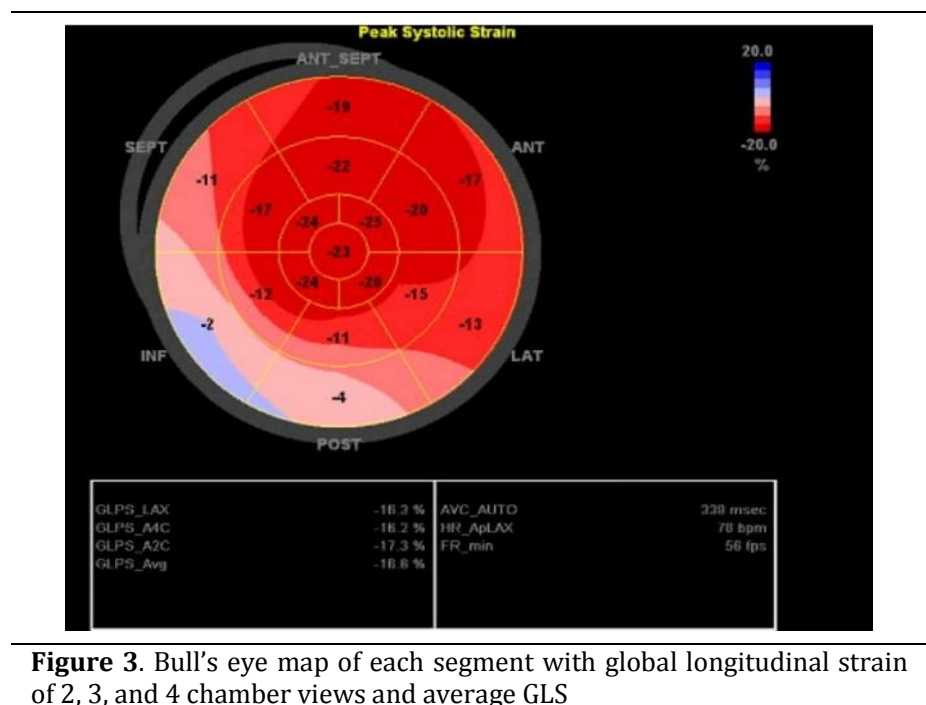

of 2,3 , and 4 chamber views and average GLS

\subsection{Echocardiography assessment}

All the patients underwent 2D echocardiography and tissue Doppler imaging (TDI) using the speckle tracking method to assess the LV function. LVEF was measured at least three times using the biplane Simpson method and the mean value was determined for each patient.

Transthoracic echocardiography evaluations were performed by a fellowship of echocardiography with at least 5 years of experience in echocardiography who were kept blind to the patients' clinical details. In the GLS evaluation, 2-chamber, 3-chamber, and 4chamber views were taken from each patient using speckle tracking (Figure 1). The images were taken digitally in three successive beats and analyzed offline using the automated function imaging (AFI) mode of the GE software. The LV contour was traced and the software automatically evaluated the longitudinal strain of each myocardial segment. Peak systolic longitudinal strain values of LAX (long axis), A2C (Apical 2 chamber), A4C (Apical 4 chamber), and average GLS were recorded (Figure 2). LV diastolic function and their indices were evaluated as well.

\subsection{Statistical analysis}

The statistical analyses were carried out in SPSS software (Version 22). Data were analyzed using descriptive statistics, including frequency distribution tables, diagrams, and measures of central tendency (mean) and dispersion (range and standard deviation). The normal distribution of the data was assessed using the Kolmogorov-Smirnov test and the homogeneity of variances using Levene's test. The significance of differences was assessed using the independent twosample t-test or its non-parametric equivalent (MannWhitney's test), as well as the Chi-square test. The relationship between the quantitative variables was assessed using Pearson's correlation coefficient. The level of statistical significance was set at 0.05 .

\section{Results}

Table 1 presents the demographic details of the participants in both case and control groups. The mean $\pm S D$ age of participants in the control and case groups was $38.79 \pm 11.74$ and $41.15 \pm 67.86$ years, respectively, which showed no significant difference $(\mathrm{P}=0.409)$. No significant differences were observed 
between the two groups in terms of the frequency of gender, body mass index (BMI), and body surface area (BSA) either $(\mathrm{P}>0.05)$.

Table 2 presents the clinical details and medical history of the patients. The disease was controlled in all the cases $(n=30)$ and no acute illness was observed. The mean duration of the disease was $6.53 \pm 7.54$ years (ranging from 3 to 33 years). No significant differences were found between the $\mathrm{DM} / \mathrm{PM}$ patients and the healthy participants in the control group in terms of end-systolic volume, heart rate, and pulmonary artery pressure values $(\mathrm{P}>0.05)$. Meanwhile, end-diastolic volume, EF, stroke volume, and cardiac output values were significantly lower in the DM/PM patients, compared to the controls $(\mathrm{P}<0.01)$. Although the patients had normal LVEF, absolute values of GLS (LAX), GLS (A4C), GLS (A2C), and average GLS (2D) were significantly lower in the patients, compared to the controls $(\mathrm{P}<0.01)$ (Table 3$)$. Furthermore, normal diastolic dysfunction and grade-one diastolic dysfunction was observed in 23 (76.7\%) and seven cases $(23.3 \%)$, respectively. Diastolic dysfunction was significantly correlated with the increased age of the patient and the duration of the disease $(\mathrm{P}<0.0001$ and $\mathrm{P}<0.01$, respectively).

No significant relationships were observed between GLS (2D) and such factors as age, gender,

\begin{tabular}{lccc}
\hline \multicolumn{2}{l}{ Table 1. Baseline characteristics of case and control groups } & & Control (n=40) \\
\hline Specifications & Case (n=30) & $38.79 \pm 11.74(17-61)$ & P-value \\
\hline Age (year) & $41.67 \pm 15.86(17-69)$ & $37(92.5 \%)$ & 0.409 \\
Female & $26(86.7 \%)$ & $3(7.5 \%)$ & 0.452 \\
Male & $4(13.3 \%)$ & $26.05 \pm 6.73$ & 0.452 \\
BMI $\left(\mathbf{K g} / \mathbf{m}^{2}\right)$ & $28.56 \pm 5.64$ & $(17.58-42.42)$ & 0.165 \\
\multirow{2}{*}{ BSA $\left(\mathbf{m}^{2}\right)$} & $(20.17-43.56)$ & $1.71 \pm 0.16$ & 0.059 \\
\hline
\end{tabular}

Note: BMI: Body Mass Index; BSA: Body Surface Area

- Data are presented as No. (\%) or Mean \pm SD (Min-Max)

\begin{tabular}{|c|c|c|c|}
\hline Variable & & Frequency & Percentage \\
\hline \multirow{3}{*}{ Underlying Disease } & DM & 5 & 16.7 \\
\hline & HTN & 4 & 13.3 \\
\hline & DLP & 2 & 6.7 \\
\hline \multirow[t]{2}{*}{ Smoking } & & 2 & 6.7 \\
\hline & $3 / 5$ & 3 & 10 \\
\hline \multirow[t]{2}{*}{ Muscle Force } & $4 / 5$ & 11 & 36.7 \\
\hline & $5 / 5$ & 16 & 53.3 \\
\hline \multirow{6}{*}{ Systemic conflict } & Systemic Symptoms & 18 & 60 \\
\hline & Dermatological manifestations & 9 & 30 \\
\hline & Gastrointestinal Symptoms & 7 & 23.3 \\
\hline & Pulmonary Involvement & 9 & 30 \\
\hline & Ophthalmic muscle involvement & 1 & 3.3 \\
\hline & Musculoskeletal involvement & 18 & 60 \\
\hline \multirow{6}{*}{ Drugs used } & MTX & 11 & 36.7 \\
\hline & Immunosuppressive (azathioprine / mycophenolate) & $11 / 6$ & $36.7 / 20$ \\
\hline & IVIG & 10 & 33.3 \\
\hline & Rituximab & 2 & 6.7 \\
\hline & Prednisolone & 25 & 83.3 \\
\hline & Discontinued medication & 1 & 3.3 \\
\hline
\end{tabular}

DM: Diabetes mellitus; HTN: Hypertension; DLP: Dyslipidemia; MTX: Methotrexate; IVIG: Intravenous immunoglobulin

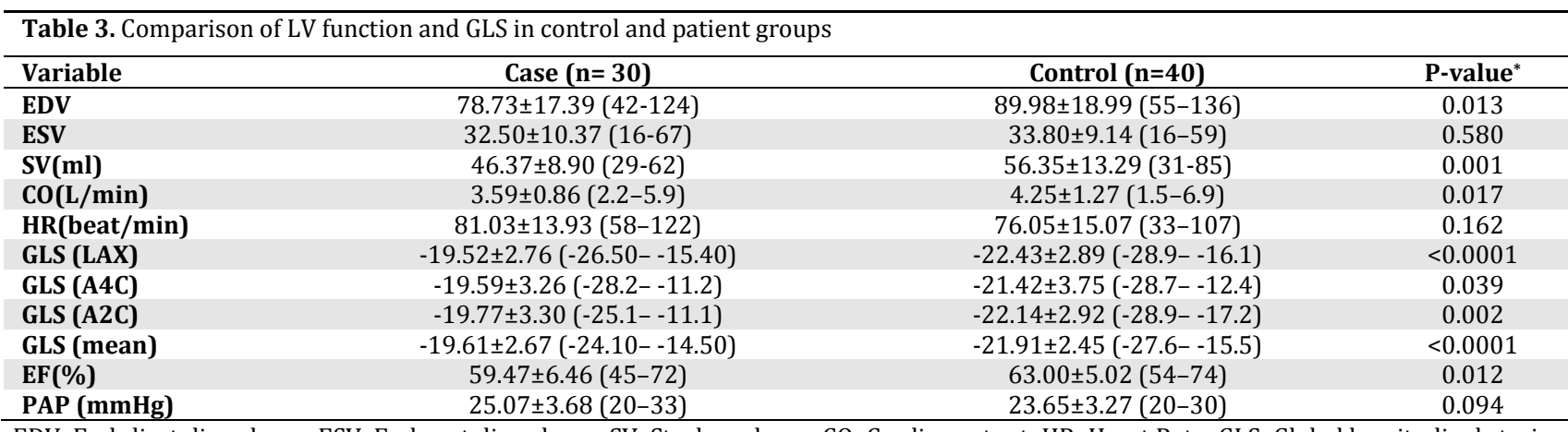

EDV: End-diastolic volume; ESV: End-systolic volume; SV: Stroke volume; CO: Cardiac output; HR: Heart Rate; GLS: Global longitudinal strain; LAX: Apical long axis; A4C: Apical 4 chamber; A2C: Apical 2 chamber; LVED: Left ventricular end systolic diameter; LVED: Left ventricular end-diastolic diameter; EF: Ejection fraction; PAP: Pulmonary arterial pressure.

-All the variables are presented as Mean \pm standard deviation (Min-Max). P-values less than (P $\leq 0.05)$ are considered statistically significant. 
comorbidity, smoking, duration of disease, BMI, BSA, use of various medications, various systemic problems, and diastolic dysfunction and muscular force $(\mathrm{P}>0.05)$.

\section{Discussion}

Cardiac problems occur in DM/PM patients and constitute one of the main causes of death in these patients $(2,18)$. Therefore, early diagnosis and treatment are essential to the patients' recovery and survival (2). The STE as a non-invasive technique was adopted in this study to evaluate GLS (which is a new variable for the evaluation of myocardial deformities) for the diagnosis of subclinical LV systolic dysfunction in DM/PM patients.

The results indicated that although LVEF was within the normal range, GLS (2D) values were significantly lower in the DM/PM patients, compared to the control group. Therefore, the present findings confirm the role of 2D STE in the early diagnosis of LV dysfunction in DM/PM patients.

Few studies have been conducted on LV dysfunction in DM/PM patients using STE and GLS evaluation. In a recent study performed by Zhong et al., cardiac function in idiopathic DM/PM patients was investigated using three-dimensional STE (3D STE). Their results showed significantly lower LV and RV GLS values in the DM/PM patients, compared to the control group (19), which is in line with the results of the present study. Another study on LV systolic dysfunction in young patients with DM also showed significantly lower GLS values in these patients, compared to the healthy controls. The results obtained by Guerra et al. showed similar systolic and diastolic dysfunctions in both the patients and controls, although GLS was significantly lower in the IIM patients, compared to the controls (20), which is consistent with the results obtained in the present study.

In both the present and the aforementioned studies, LV systolic dysfunction was observed using GLS even in the patients with normal LVEF, which is indicative of the fact that GLS is a valuable marker for the assessment of subclinical cardiac problems. Currently, some researchers consider LVEF dysfunction to be the last marker of cardiac damage.

Therefore, variables of conventional echocardiography by the 2D method are probably not enough for the identification of subclinical cardiac problems. An early presentation of cardiac involvement, especially in the preclinical stage, is possible through the evaluation of $\mathrm{LV}$ diastolic dysfunction using TDI (21). Peter et al. reported LV dysfunction following the diagnosis of DM/PM in 30 people with no known cardiac symptoms using TDI analysis instead of LVEF assessment (22). GLS may be more reliable than biplane LVEF since it is angleindependent and automatically calculated by the software $(17,23)$.

Many other studies, including studies conducted by Fine et al. (24), Bakoum et al. (25), and Poorzand et al. (26) have shown that the GLS evaluation of the LV using STE can reveal LV systolic and diastolic dysfunctions in patients with the rheumatological disorder, such as lupus and rheumatoid arthritis with no history of heart disease.

According to the present findings and the results of similar studies, GLS evaluation can detect DM/PM dysfunction with no cardiac symptoms or risk factors. Therefore, STE is a valuable technique for the diagnosis of subclinical LV dysfunction and is superior to conventional 2D echocardiography.

In this study, no significant relationship was observed between the measured LV systolic dysfunction and such factors as the patients' demographic and clinical details, duration of the disease, medications used, and different systemic disorders. However, LV diastolic dysfunction was significantly correlated with both increased patient age and increased duration of the disease. The correlation of diastolic dysfunction impairment with age has been reported in different studies (27-29). The findings of the present study were consistent with the previous study which reported a significant association between the degree of $\mathrm{LV}$ diastolic dysfunction and duration of DM due to the subclinical involvement of myocardium with the development of the inflammatory process (30). In addition, it is reported that the increased duration of the disease is often correlated with increased cell damage, pathological calcification, and increased inflammatory cytokines, such as tumor necrosis factor- $\alpha$ which affect diastolic function in DM (31-33).

Cardiac damage in DM/PM patients is a long-term process and the different incidence of cardiac involvement in various studies can be due to different definitions of cardiac abnormalities in these studies. All cardiac structures, such as heart valves, conduction system, myocardium, endocardium, pericardium, and pulmonary and coronary arteries can be affected by PM/DM (34) and cardiac involvement may include myocarditis, coronary artery disease, angina, arrhythmias $(35,36)$. Some studies have been conducted to assess LV diastolic function in DM and PM patients using echocardiography techniques, such as TDI and conventional echocardiography. These studies reported a high prevalence of LV diastolic dysfunction in DM/PM patients, compared to controls $(22,30)$.

Regarding the limitations of the present study, one can refer to the small sample size.

\section{Conclusion}

Although the examined patients in this study had normal LVEF values, LV systolic dysfunction 
diagnosed by GLS was significantly higher in these DM/PM patients with no known heart disorders, compared to the controls. Therefore, new STE techniques can be used for the diagnosis of subclinical disorders in LV systolic function. The early diagnosis of systolic dysfunction using GLS can help prevent clinically symptomatic heart failure which in turn leads to the early management and treatment of these patients. Further prospective studies on the long-term prognosis of these patients seem to be necessary. However, subclinical systolic dysfunctions have been reported in some studies. Moreover, regular 2D echocardiography evaluation is important for the detection of cardiac problems in these patients and should not be a procedure limited to patients in advanced stages or with longer durations of the disease.

\section{Acknowledgments}

The authors would like to thank all colleagues in the echocardiography ward of Imam Khomeini Hospital and the staff of rheumatology polyclinics in Ahvaz Jundishapur University of Medical Sciences, Ahvaz, Iran.

\section{Footnotes}

Author's contributions: Dr. Nehzat Akiash and Dr. Elham Rajaei designed the work. Dr. Nasibeh Akiash, Dr. Nehzat Akiash, and Dr. Shirin Azizidoost collected data, prepared and designed the figures. Dr. Karim Mowla, Dr. Elham Rajaei, and Dr. Nehzat Akiash were involved in data analysis and interpretation. Dr. Karim Mowla and Dr. Elham Rajae helped with the critical revision of the article. Dr. Nehzat Akiash, Dr.Shirin Azizidoost, and Dr. Nasibeh Akiash drafted the manuscript.

Conflicts of Interest: The authors declare that they have no conflict of interest regarding the publication of the present study.

Funding/Support: This work was financially supported by Ahvaz Jundishapur University of Medical Sciences, Ahvaz, Iran (Grant number: CVRC9606).

Ethical Approval: The study protocol was approved by the Ethics Committee of Ahvaz Jundishapur University of Medical Sciences, Ahvaz, Iran (IR.AJUMS.REC.1396.1004).

Informed Consent: The informed written consent was obtained from the participants in this study.

\section{References}

1. Dimachkie MM, Barohn RJ, Amato AA. Idiopathic inflammatory myopathies. Neurol Clin. 2014;32(3):595-628. doi: 10.1016/j.ncl.2014.04.007. [PubMed: 25037081].

2. Oldroyd A, Lilleker J, Chinoy H. Idiopathic inflammatory myopathies-a guide to subtypes, diagnostic approach and treatment. Clin Med. 2017;17(4):322-8. doi: 10.7861/ clinmedicine.17-4-322. [PubMed: 28765407].

3. Tanboon J, Nishino I. Classification of idiopathic inflammatory myopathies: pathology perspectives. Curr Opin Neurol. 2019;32(5):704-14. doi: 10.1097/WCO.0000000000000740. [PubMed: 31369423].

4. Mastaglia FL, Phillips BA. Idiopathic inflammatory myopathies: epidemiology, classification, and diagnostic criteria. Rheum Dis Clin North Am. 2002;28(4):723-41. doi: 10.1016/s0889857x(02)00021-2. [PubMed: 12510664].

5. Lundberg IE, Miller FW, Tjärnlund A, Bottai M. Diagnosis and classification of idiopathic inflammatory myopathies. J Intern Med. 2016;280(1):39-51. doi: 10.1111/joim.12524. [PubMed: 27320359]

6. Dalakas MC, Hohlfeld R. Polymyositis and dermatomyositis. Lancet. 2003;362(9388):971-82. doi: 10.1016/S01406736(03)14368-1. [PubMed: 14511932].

7. Na SJ, Kim SM, Sunwoo IN, Choi YC. Clinical characteristics and outcomes of juvenile and adult dermatomyositis. J Korean Med Sci. 2009;24(4):715-21. doi: 10.3346/jkms.2009.24.4.715. [PubMed: 19654958].

8. Diederichsen L, Simonsen J, Diederichsen A, Hvidsten S, Hougaard M, Junker P, et al. Cardiac abnormalities in adult patients with polymyositis or dermatomyositis as assessed by noninvasive modalities. Arthrit Care Res. 2016;68(7):1012-20. doi: 10.1002/acr.22772. [PubMed: 26502301].

9. Gupta R, Wayangankar SA, Targoff IN, Hennebry TA. Clinical cardiac involvement in idiopathic inflammatory myopathies: a systematic review. Int J Cardiol. 2011;148(3):261-70. doi: 10.1016/j.ijcard.2010.08.013. [PubMed: 20826015].

10. Gazeley DJ, Cronin ME. Diagnosis and treatment of the idiopathic inflammatory myopathies. Ther Adv Musculoskelet Dis.2011;3(6):315-24. doi: 10.1177/1759720X11415306. [PubMed: 22870489].

11. Chen F, Peng Y, Chen M. Diagnostic approach to cardiac involvement in idiopathic inflammatory myopathies. Int Heart J. 2018;59(2):256-62. doi: 10.1536/ihj.17-204. [PubMed: 29563381].

12. Blessberger $\mathrm{H}$, Binder $\mathrm{T}$. Non-invasive imaging: two dimensional speckle tracking echocardiography: basic principles. Heart 2007;96(9):716-22. doi: 10.1136/hrt.2007.141002. [PubMed: 20424157].

13. Geyer H, Caracciolo G, Abe H, Wilansky S, Carerj S, Gentile F, et al. Assessment of myocardial mechanics using speckle tracking echocardiography: fundamentals and clinical applications. $\mathrm{J} \mathrm{Am}$ Soc Echocardiogr. 2010;23(4):351-69. doi: 10.1016/j.echo. 2010.02.015. [PubMed: 20362924]

14. Spethmann S, Dreger H, Schattke S, Riemekasten G, Borges AC, Baumann G, et al. Two-dimensional speckle tracking of the left ventricle in patients with systemic sclerosis for an early detection of myocardial involvement. Eur Heart J Cardiovasc Imaging. 2012;13(10):863-70. doi: 10.1093/ehjci/jes047. [PubMed: 22427402].

15. Diniz MD, Kozu KT, Elias AM, Lianza AC, Sawamura KS Menezes CR, et al. Echocardiographic study of juvenile dermatomyositis patients: new insights from speckle-trackingderived strain. Clin Rheumatol. 2021;40(4):1497-505. doi: 10.1007/s10067-020-05418-4. [PubMed: 32989504].

16. Zhang L, Wang GC, Ma L, Zu N. Cardiac involvement in adult polymyositis or dermatomyositis: a systematic review. Clin Cardiol. 2012;35(11):686-91. doi: 10.1002/clc.22026. [PubMed: 22847365].

17. Karlsen S, Dahlslett T, Grenne B, Sjøli B, Smiseth O, Edvardsen $\mathrm{T}$, et al. Global longitudinal strain is a more reproducible measure of left ventricular function than ejection fraction regardless of echocardiographic training. Cardiovasc Ultrasound. 2019;17(1):18. doi: 10.1186/s12947-019-0168-9. [PubMed: 31477137].

18. Lundberg I. The heart in dermatomyositis and polymyositis. Rheumatology. 2006;45(Suppl 4):iv18-21. doi: 10.1093/ rheumatology/kel311. [PubMed: 16980718].

19. Zhong Y, Bai W, Xie Q, Sun J, Tang H, Rao L. Cardiac function in patients with polymyositis or dermatomyositis: a threedimensional speckle-tracking echocardiography study. Int $J$ Cardiovasc Imaging. 2018;34(5):683-93. doi: 10.1007/s10554- 
017-1278-9. [PubMed: 29168054].

20. Guerra F, Gelardi C, Capucci A, Gabrielli A, Danieli MG. Subclinical cardiac dysfunction in polymyositis and dermatomyositis: a speckle-tracking case-control study. $J$ Rheumatol. 2017;44(6):815-21. doi: 10.3899/jrheum.161311. [PubMed: 28365571].

21. Lu Z, Wei Q, Ning Z, Qian-Zi Z, Xiao-Ming S, Guo-Chun W. Left ventricular diastolic dysfunction--early cardiac impairment in patients with polymyositis/dermatomyositis: a tissue Doppler imaging study. J Rheumatol. 2013;40(9):1572-7. doi: 10.3899/jrheum.130044. [PubMed: 23908445].

22. Péter A, Balogh Á, Szilágyi S, Faludi R, Nagy-Vincze M, Édes I, et al. Echocardiographic abnormalities in new-onset polymyositis/dermatomyositis. J Rheumatol. 2015;42(2):27281. doi: 10.3899/jrheum.140626. [PubMed: 25433528].

23. Plana JC. Detection by cardiac imaging: LV ejection fraction, diastolic dysfunction, tissue doppler, and speckle tracking echocardiography. Anti-cancer treatments and cardiotoxicity. Massachusetts: Academic Press; 2017. P. 273-9. doi: 10.1016/B978-0-12-802509-3.00023-6.

24. Fine NM, Crowson CS, Lin G, Oh JK, Villarraga HR, Gabriel SE. Evaluation of myocardial function in patients with rheumatoid arthritis using strain imaging by speckle-tracking echocardiography. Ann Rheum Dis. 2014;73(10):1833-9. doi: 10.1136/annrheumdis-2013-203314. [PubMed: 23873875].

25. Bakhoum SW, El Basel M, Alnaggar AR, Hamdy MS, Hussein H. Assessment of left ventricular function in systemic lupus erythematosus patients by speckle tracking echocardiography: Relation to circulating endothelial progenitor cells. Egypt Rheumatol. 2015;37(4):S33-41. doi: 10.1016/j.ejr.2015.05.002.

26. Poorzand H, Mirfeizi SZ, Javanbakht A, Alimi H. Comparison of echocardiographic variables between systemic lupus erythematosus patients and a control group. Arch Cardiovasc Imaging. 2015;3(2):e30009. doi: 10.5812/acvi.30009.

27. Kuznetsova T, Herbots L, López B, Jin Y, Richart T, Thijs L, et al. Prevalence of left ventricular diastolic dysfunction in a general population. Circ Heart Fail. 2009;2(2):105-12. doi: 10.1161/CIRCHEARTFAILURE.108.822627. [PubMed: 19808325].

28. Han L, Bai X, Lin H, Sun X, Chen X. Gender differences in the relationship between age-related carotid intima-media thickness and cardiac diastolic function in a healthy Chinese population. J Card Fail. 2013;19(5):325-32. doi: 10.1016/j.cardfail.2013.03.012. [PubMed: 23663815].

29. Salmasi AM, Alimo A, Jepson E, Dancy M. Age-associated changes in left ventricular diastolic function are related to increasing left ventricular mass. Am J Hypertens. 2003; 16(6):473-7. doi: 10.1016/s0895-7061(03)00846-x. [PubMed: 12799096].

30. Wang H, Liu HX, Wang YL, Yu XQ, Chen XX, Cai L. Left ventricular diastolic dysfunction in patients with dermatomyositis without clinically evident cardiovascular disease. J Rheumatol. 2014;41(3):495-500. doi: 10.3899/jrheum.130346. [PubMed: 24429180].

31. Kim E, Cook-Mills J, Morgan G, Sredni ST, Pachman LM. Increased expression of vascular cell adhesion molecule 1 in muscle biopsy samples from juvenile dermatomyositis patients with short duration of untreated disease is regulated by miR-126. Arthritis Rheum. 2012;64(11):3809-17. doi: 10.1002/art.34606. [PubMed: 22740338].

32. Pachman LM, Abbott K, Sinacore JM, Amoruso L, Dyer A, Lipton $\mathrm{R}$, et al. Duration of illness is an important variable for untreated children with juvenile dermatomyositis. J Pediatr. 2006;148(2):247-53. doi: 10.1016/j.jpeds.2005.10.032. [PubMed: 16492437].

33. Zhao Y, Fedczyna TO, McVicker V, Caliendo J, Li H, Pachman LM. Apoptosis in the skeletal muscle of untreated children with juvenile dermatomyositis: impact of duration of untreated disease. Clin Immunol. 2007;125(2):165-72. doi: 10.1016/j.clim. 2007.06.011. [PubMed: 17704000]

34. Liu XH, Feng XJ, Shi JY, Jia FW, Liu YX, Zhu YL, et al. The quest for diagnostic approaches of cardiac involvement in polymyositis and dermatomyositis. Ann Palliat Med. 2020;9(4):2256-70. doi: 10.21037/apm-19-650. [PubMed: 32648461].

35. Yang SH, Chang C, Lian ZX. Polymyositis and dermatomyositischallenges in diagnosis and management. J Transl Autoimmun. 2019;2:100018. doi: 10.1016/j.jtauto.2019.100018. [PubMed: 32743506].

36. Huber AT, Bravetti M, Lamy J, Bacoyannis T, Roux C, de Cesare $A$, et al. Non-invasive differentiation of idiopathic inflammatory myopathy with cardiac involvement from acute viral myocarditis using cardiovascular magnetic resonance imaging T1 and T2 mapping. I Cardiov Magn Reson. 2018;20(1):11. doi: 10.1186/s12968-018-0430-6. [PubMed: 29429407]. 